\title{
LOBIVIA FEROX BRITTON ET ROSE (CACTACEAE), NUEVO REGISTRO PARA LA FLORA CHILENA
}

\author{
LOBIVIA FEROX BRITTON ET ROSE (CACTACEAE), NEW RECORD FOR \\ THE CHILEAN FLORA
}

\author{
Raquel Pinto B. ${ }^{1}$
}

\begin{abstract}
RESUMEN
Se registra por primera vez Lobivia ferox Britton et Rose (Cactaceae) en el altiplano de Tarapacá, norte de Chile, constituyendo una extensión de rango para esta especie. Se detalla la descripción de la especie y aspectos fenológicos y ecológicos.

Palabras Claves: Cactaceae, Lobivia ferox, nuevo registro, flora chilena.
\end{abstract}

\section{INTRODUCCION}

En septiembre del año 2000 la Ilustre Municipalidad de Iquique trasladó 100 ejemplares de Echinopsis atacamensis (Phil.) Freidrich et G. D. Rowley desde el altiplano chileno, localidad de Panavinto (3.800 m), al parque de Playa Cavancha en la costa. Viajamos a Panavinto para conocer el lugar de donde se extrajeron los cactos y registrar el daño ecológico resultante. Encontramos para gran sorpresa nuestra una hermosa comunidad de alrededor de 3.000 ejemplares. Entre ellos numerosos juveniles. En esta comunidad nos llamó la atención la presencia de un cactus esférico de largas espinas

${ }^{1}$ Equipo de Estudios de Ecosistemas de Niebla. Dalmacia 3251, Iquique, Chile. E-mail: raquelpinto@vtr.net

\begin{abstract}
Lobivia ferox Britton et Rose (Cactaceae) is a new record for the Chilean flora, expanding the known range of distribution of this species to the altiplano of Tarapacá, northern Chile. This study includes a description of the species and phenological and ecological observations.
\end{abstract}

KeYwords: Cactaceae, Lobivia ferox, new record, Chilean flora.

y que no correspondía a ninguno de los registrados en las tierras altas del norte de Chile. Se trataba de Lobivia ferox, un nuevo registro para la flora chilena, por lo que decidimos iniciar su estudio.

El género Lobivia fue descrito por Britton et Rose en 1922. Pertenece a la familia Cactaceae, subfamilia Cereoidae, tribu Trichocereae (F. Buxb.). El nombre Lobivia es un anagrama de Bolivia, por ser un grupo típico de ese país. El límite entre los géneros Lobivia, Echinopsis y Trichocereus es difícil de definir. Actualmente hay una tendencia a aglutinar todo bajo el género Echinopsis. Se acepta la inclusión de Trichocereus en Echinopsis, así como también Lobivia en Echinopsis, lo que de acuerdo a los botánicos Roberto Kiesling y Roberto Vásquez es discutible (comunicación personal). El género habita las tierras altoandinas de Perú, Bolivia y Argentina. En la actualidad Brako y Zarucchi (1993) citan 33 especies y 16 variedades para Perú, Kiesling (1999) cita 18 especies para Bolivia y 23 especies 


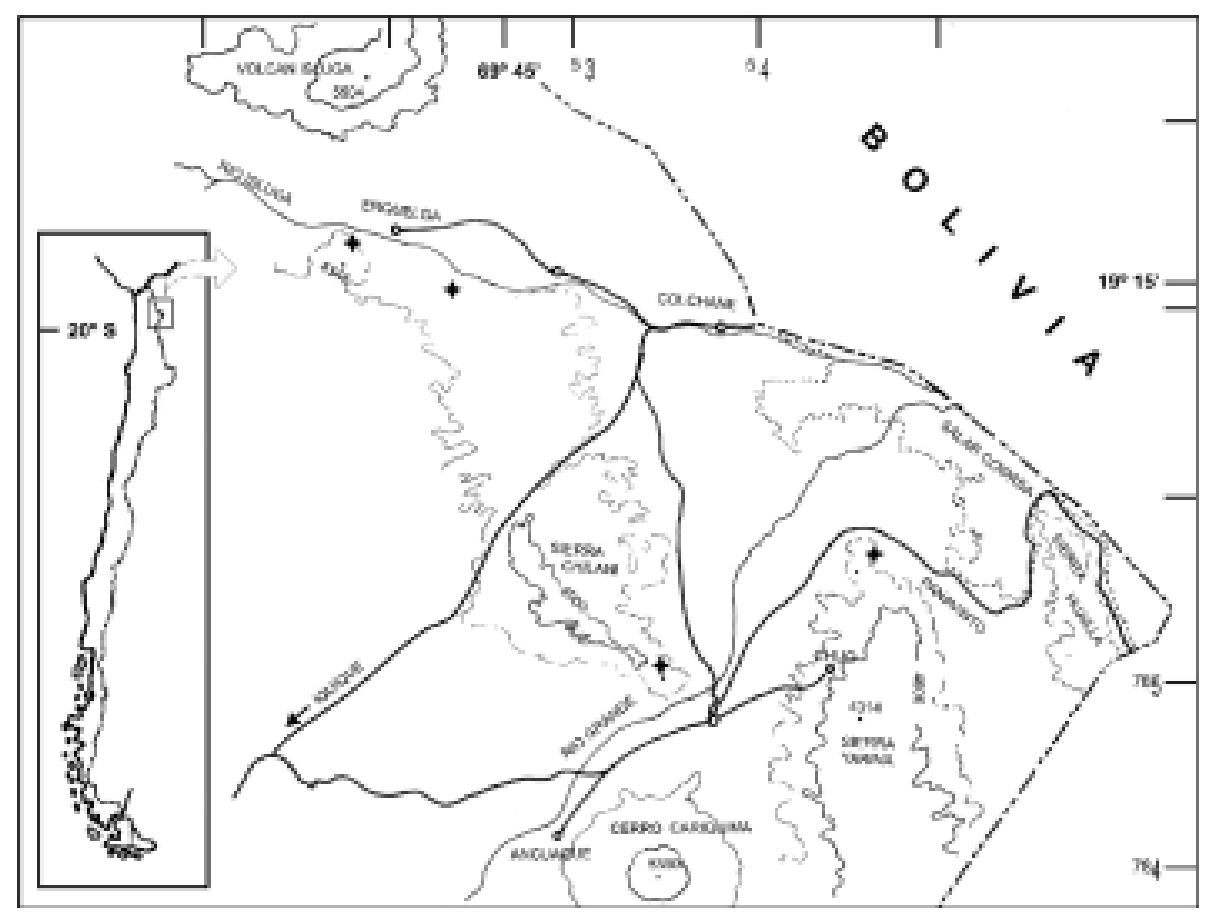

Figura 1. Localización de los sectores donde se detectó la presencia de Lobivia ferox, en el altiplano de Iquique. * Sitios de estudio.
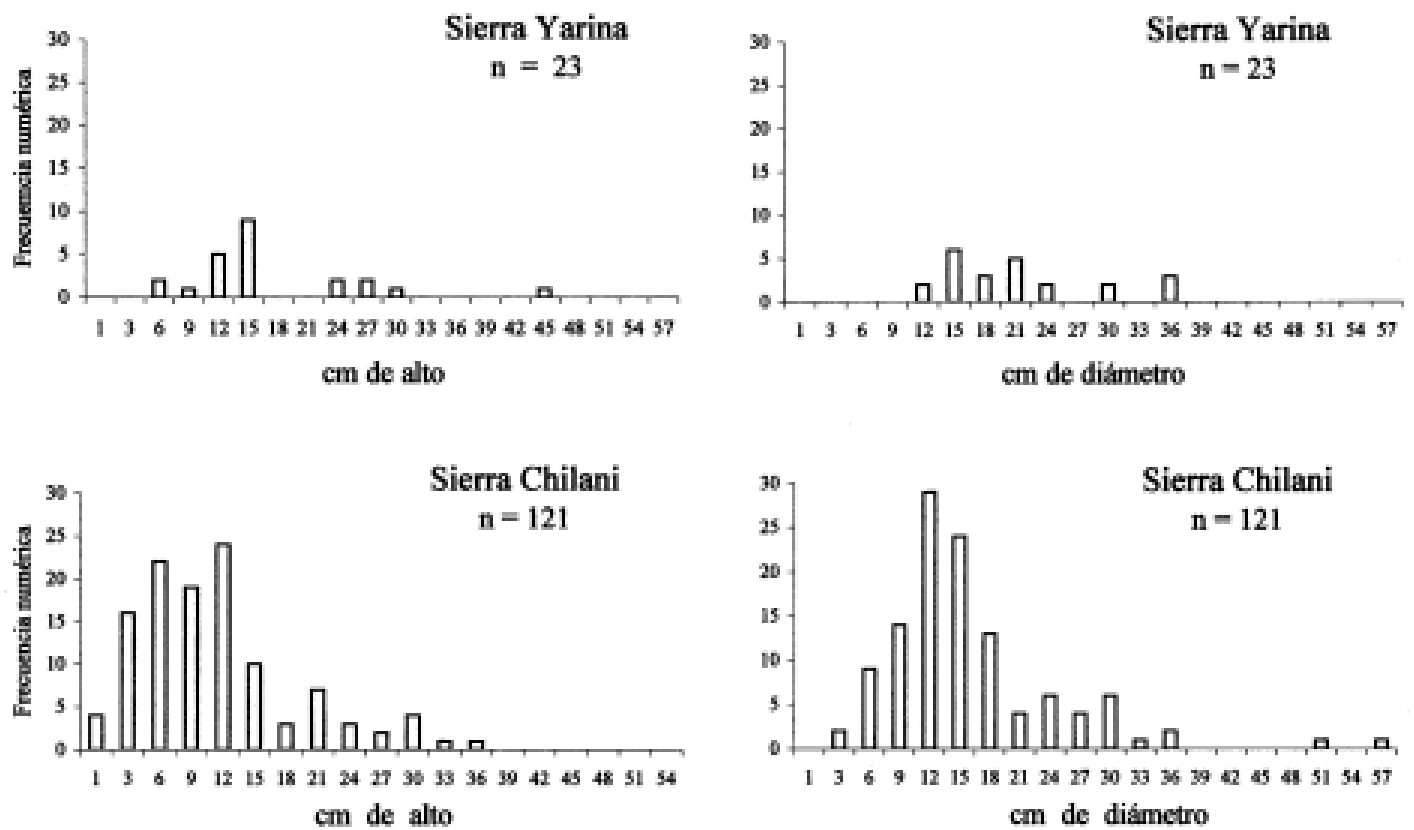

FIgura. 2. Distribución altitudinal de la densidad de Lobivia ferox en el sector altoandino de la región de Tarapacá. 
para Argentina. En el checklist de las cactáceas aparecen 190 especies de Lobivia (Hunt 1992).

Descripción del género Lobivia (adaptado de Britton et Rose, Cact. 3:49. 1922): Planta globular a cortamente cilíndrica. Simple o agrupada. Costillas con muchas espinas. Flores diurnas, en forma de embudo corto a acampanadas, de disposición lateral, se originan en areolas antiguas; en algunas especies cerca del ápice y en otras por los lados de la planta. Tubo floral corto y ancho. Flor roja en la especie típica, pero en otras amarillas o blancas. Escamas del ovario generalmente con pelos largos en las axilas. Fruto pequeño, globular. La característica de este género es la forma de la flor con presencia de un denso anillo de pelos dentro del tubo floral, bajo los estambres.

Descripción de Lobivia ferox (Britton et Rose 1922): Planta globular de $30 \mathrm{~cm}$ de diámetro o más. De raíces fibrosas. Costillas numerosas, a menudo 29 o más, muy onduladas y quebradas en finos y agudos tubérculos de 2 a $3 \mathrm{~cm}$ de largo. Espinas café claro, algunas veces moteadas. 10 a 12 espinas radiales, delgadas de 4 a $6 \mathrm{~cm}$ de largo algo curvadas. 3 ó 4 espinas centrales curvadas hacia arriba, de 10 a $15 \mathrm{~cm}$ de largo, algo aplanadas en hileras verticales más bien débiles. Botones lanudos. Flores y frutos no vistos.

La especie fue descrita por $\mathrm{Br}$. et $\mathrm{R}$. en base a una planta que fue colectada en los cerros al este de Oruro, Bolivia, en 1914. Posteriormente Curt Backeberg la transfirió al género Pseudolobivia (Backeb.) Backeb., quedando como P. ferox (Britton et Rose) Backeb., Stachlige Wildnis:219, 1942. Pero en 1975 Walter Rausch, el más grande especialista en Lobivia, la mantuvo como L. ferox. En 1985 Rausch publica una segunda revisión de este género.

Sinónimos: Lobivia longispina Britton et Rose.; L. ferox var. camargensis Ritter; L. ducispauli Fric.; Echinopsis longispina (Br. et R.) Backeb.; E. ferox Backeb.; E. lecoriensis Cárdenas

Variedades: L. ferox var. longispina (Britton et Rose.) Rausch y L. ferox var. potosina (Werderm.) Rausch.

Distribución geográfica: Extremo nor oeste de Argentina ( $25^{\circ} 91^{\prime} S$; $65^{\circ} 20^{\prime} \mathrm{W}$ ) en Salta y Jujuy (por sobre los $2.000 \mathrm{~m}$ de altitud), hasta Bolivia $\left(18^{\circ} \mathrm{S} ; 67^{\circ} \mathrm{W}\right)$ en el Departamento de Oruro.

\section{METODOS}

ÁREA DE ESTUDIO: El estudio se realizó en noviembre del 2001 y febrero del 2002, en el altiplano de la I Región de Tarapacá, norte de Chile, a 300 km al interior de Iquique por sobre $10 \mathrm{~s} 3.700 \mathrm{~m}$ de altitud (Fig. 1). Por conversaciones con pastores aymaras logramos definir cuatro sectores con presencia de L. ferox: 1. Sierra de Yarina, frente al Salar de Coipasa, Panavinto, Cariquima. 2. Sierra Chilani, al noroeste de Cariquima (Fig. 2). 3. Cerro Coraguane, quebrada de Arabilla y 4. Este de Enquelga, río Isluga, comuna de Colchane.

Se midió densidad en Sierra de Yarina y Sierra Chilani en 4 y 5 cuadrantes de $10 \times 10 \mathrm{~m}$ a lo largo de un transecto altitudinal. Se registró la estructura de talla de la población midiendo alto y diámetro de las plantas. Se registró también el estado de la población, contando número de ejemplares muertos.

Los ejemplares fueron depositados en el herbario de la Universidad de Concepción. Se examinaron numerosos ejemplares observando detenidamente sus características, con el propósito de conseguir una descripción detallada de la especie aportando nuevos antecedentes. Con los cortes para herbario se tuvo gran dificultad, ya que esta especie presenta un tejido acuoso que se oxida fácilmente adquiriendo un color anaranjado a negruzco.

\section{Material estudiado}

CHILE, I Región, Provincia de Iquique:

Comuna de Colchane, Cerro Pacocollo, Sierra de Yarina $19^{\circ} 23^{\prime} \mathrm{S}-68^{\circ} 33^{\prime} \mathrm{W}, 3710 \mathrm{~m}, 2-11-01$, PINTO \& KIRBERG, $\mathrm{N}^{\circ} 15529310$ (CONC).

Cerro Jilimani, Sierra Chilani, $19^{\circ} 26^{\prime}$ S $-68^{\circ} 40^{\prime} \mathrm{W}$, 3794 m, 2-02-02, PINTO \& KIRBERG, $\mathrm{N}^{\circ} 155291$ 16, 15529411,1552951213 (CONC).

Sierra de Huaillas, junto a Equinopsis atacamensis en la comuna de Colchane ( $\left.19^{\circ} 21^{`} \mathrm{~S} ; 68^{\circ} 27^{`} \mathrm{~W}\right)$ y Quebrada de Coscaya, junto a Oreocereus leucotrichus en la comuna de Pica (19 $51^{`} \mathrm{~S} ; 69^{\circ}$ 02`W), PINTO \& KIRBERG. 
Gayana Bot. 59(2), 2002

TABLA I. Flora asociada a Lobivia ferox.

\begin{tabular}{|c|c|c|c|c|}
\hline & Familia & Especie & $\mathrm{TC}$ & Nombre comon \\
\hline \multicolumn{5}{|l|}{ Pteridophyta } \\
\hline & \multirow[t]{2}{*}{ Adiantaceae } & Cheilantes pruinata Kaulf. & hp & \\
\hline & & Notholaena nivea (Poir.) A.N. Desv. var nivea & hp & \\
\hline \multicolumn{5}{|l|}{ Angisopermatophyta } \\
\hline \multirow[t]{17}{*}{ Dicotyledoneae } & \multirow[t]{7}{*}{ Asteraceae } & Baccharis tola Phil. & ar & æаса \\
\hline & & Baccharis boliviensis (Wedd.) Cabrera & ar & \\
\hline & & Chuquirraga atacamensis $\mathrm{O} . \mathrm{K}$. & ar & \\
\hline & & Parastrephia lucida (Meyen) Cabrera & ar & tola, sipa \\
\hline & & Senecio nutans Sch. Bip. & ar & chachacoma \\
\hline & & Stevia philippiana Hieron. & ar & \\
\hline & & Tagetes multiflora H.B.K. & ha & \\
\hline & \multirow[t]{5}{*}{ Cactaceae } & Echinopsis atacamensis (Phil.) Fried. et Rowl. & sa & card n, pasakana (fruto) \\
\hline & & Lobivia ferox $\mathrm{Br}$. et $\mathrm{R}$. & $\mathrm{sb}$ & sancayo, sancave, sancahue \\
\hline & & Opuntia echinaceae (Ritter) A. Hoffmann J. & $\mathrm{sb}$ & piscaya \\
\hline & & Opuntia ignescens Vaupel & $\mathrm{sb}$ & jala-jala \\
\hline & & Opuntia soehrensii $\mathrm{Br}$. et $\mathrm{R}$. & $\mathrm{sb}$ & ayrampu \\
\hline & Papilionaceae & Adesmia spinosissima Meyen ex Vogel & ar & aæagualla \\
\hline & \multirow[t]{2}{*}{ Solanaceae } & Dunalia spinosa (Meyen.) Dammer & ar & \\
\hline & & Fabiana ramulosa (Wedd.) A.T. Hunz. et Barboz & ar & quipa \\
\hline & \multirow[t]{2}{*}{ Verbenaceae } & Acantholippia punensis Botta & ar & rica-rica \\
\hline & & Junellia seriphioides (Gill. et Hook.) Mold. & ar & \\
\hline Monocotyledoneae & Gramineae & Stipa nardoides (Phil.) Hackel ex Hitchcock & hp & \\
\hline
\end{tabular}

\section{RESULTADOS}

Descripción del hábitat: Crece en una franja altitudinal muy estrecha entre los 3.700 y $4.000 \mathrm{~m}$. Habita laderas de 20 a $30^{\circ}$ de inclinación, de orientación N, NE y E, en suelos de roca volcánica blan$\mathrm{ca}$, en grietas de rocas o en suelo abierto pedregoso. También se la encuentra bajo arbustos como Baccharis, Senecio, Fabiana. Comparte su hábitat con otras cactáceas como Echinopsis atacamensis y 3 especies de Opuntia; O. echinaceae, O. ignescens y $O$. soehrensii. La Tabla I presenta una lista de las especies registradas de flora asociada a $L$. ferox.

Densidad: Su distribución es bastante heterogénea, encontrándose sectores de baja densidad con 1 ejemplar/100 $\mathrm{m}^{2}$ en las coordenadas UTM 546682 - 7856721 a los $3.710 \mathrm{~m}$ y otros sectores de alta densidad hasta con 15 ejemplares/100 $\mathrm{m}^{2}$ en las coordenadas UTM 546232 - 7856808 a los 3.780 m en Sierra Yarina. Las mayores densidades de Lobivia (46 ejemplares $/ 100 \mathrm{~m}^{2}$ ) fueron registradas a los
$3.850 \mathrm{~m}$ en Sierra Chilani (Fig. 2). En Enquelga la densidad es menor, en la Quebrada de Arabilla se registró un promedio de 4 ejemplares $/ 100 \mathrm{~m}^{2}$ en las coordenadas UTM 518587 - 7874065 a los 3.890 m y en río Isluga 1 ejemplar $/ 100 \mathrm{~m}^{2}$ en las coordenadas UTM $522672-7872908$ a los 3.949 m.

Estado de la población: Se constató que alrededor del 70\% de la población de Lobivia está viva, de un total de 139 plantas medidas, incluyendo individuos de todas las edades.

Tamaño de la planta: La población de L. ferox muestra una distribución normal con rangos de todos los tamaños, registrándose individuos de 1 a $45 \mathrm{~cm}$ de alto. Sin embargo, la gran mayoría se encuentra entre los 10 y $15 \mathrm{~cm}$ de alto. La Fig. 3 muestra la estructura de talla de la población de Sierra Yarina y Sierra Chilani.

Descripción de la planta: Crece solitaria o formando grupos. La forma solitaria puede ser globular 

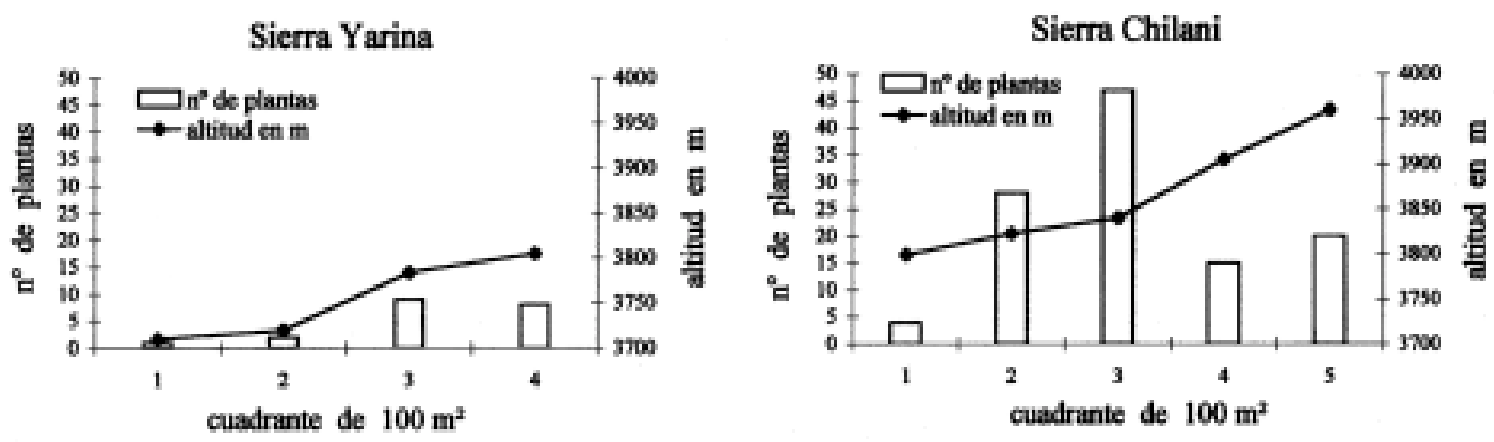

FiguRA 3. Distribución de frecuencia de tallos de Lobivia ferox

TABLA II. Variación morfológica de tallo y raíz, medido en cm.

\begin{tabular}{lcccc}
\hline \multicolumn{1}{c}{ Forma } & alto & difmetro & largo & ancho \\
\hline globular-espinuda & 18 & 16 & 26 & 2,5 \\
deprimida-pocas espinas & 7 & 14 & 20 & 6,5 \\
\hline
\end{tabular}

o deprimida. La forma agrupada la observamos en el $26 \%$ de la población en individuos de todos los tamaños. Se trata habitualmente de grupos de 2 a 9 cabezas. El ejemplar más grande registrado formaba un cojín de $55 \mathrm{~cm}$ de diámetro por $30 \mathrm{~cm}$ de alto compuesto por 20 cabezas. Esta forma se produce por gemación del tallo, ya que poseen una sola raíz. Raíz engrosada tuberosa o con ramificaciones fibrosas. Costillas numerosas (25 a 30), sesgadas, anchas (1 a $1,5 \mathrm{~cm}$ de ancho) y profundas $(1 \mathrm{~cm}$ de alto). Tubérculo agudo de $2 \mathrm{~cm}$ de largo y $1 \mathrm{~cm}$ de alto. Al corte transversal presenta una vena central de xilema ancha; así, un tallo de $16 \mathrm{~cm}$ de diámetro presenta una vena central de $10 \mathrm{~cm}$ de diámetro. Epidermis color verde azulado en la forma deprimida, que con envejecimiento va variando a tonos amarillentos y rojizos. Areolas con fieltro gris, ovaladas de $1,5 \mathrm{~cm}$ de largo por 0,6 cm de ancho. Espinas duras, hasta
18 por areola. 5 a 7 espinas centrales grandes de 7 a $12 \mathrm{~cm}$ de largo, gruesas y curvadas hacia arriba, algunas terminando en gancho. En algunos casos las largas espinas se vuelven espiraladas. 9 a 11 espinas radiales más pequeñas, finas y algunas algo horizontales de 1,5 a 3,5 cm de largo. Espinas de color gris café o amarillas blanquizcas, oscuras en la punta, rojizas al trasluz y perennes. En ejemplares adultos solitarios las espinas basales envejecen, ennegrecen y se vuelven quebradizas. El patrón más común es de formas muy espinudas (Fig. 4 B) también existen formas con pocas espinas (Fig. 4 C), lo que se presenta generalmente en los de menor tamaño y formas deprimidas. Pareciera que formas más cilíndricas presentan muchas espinas y raíces fibrosas largas y angostas y formas deprimidas presentan pocas espinas y raíz tuberosa ancha con pocas ramificaciones. La Tabla II presenta variaciones morfológicas de L. ferox. 

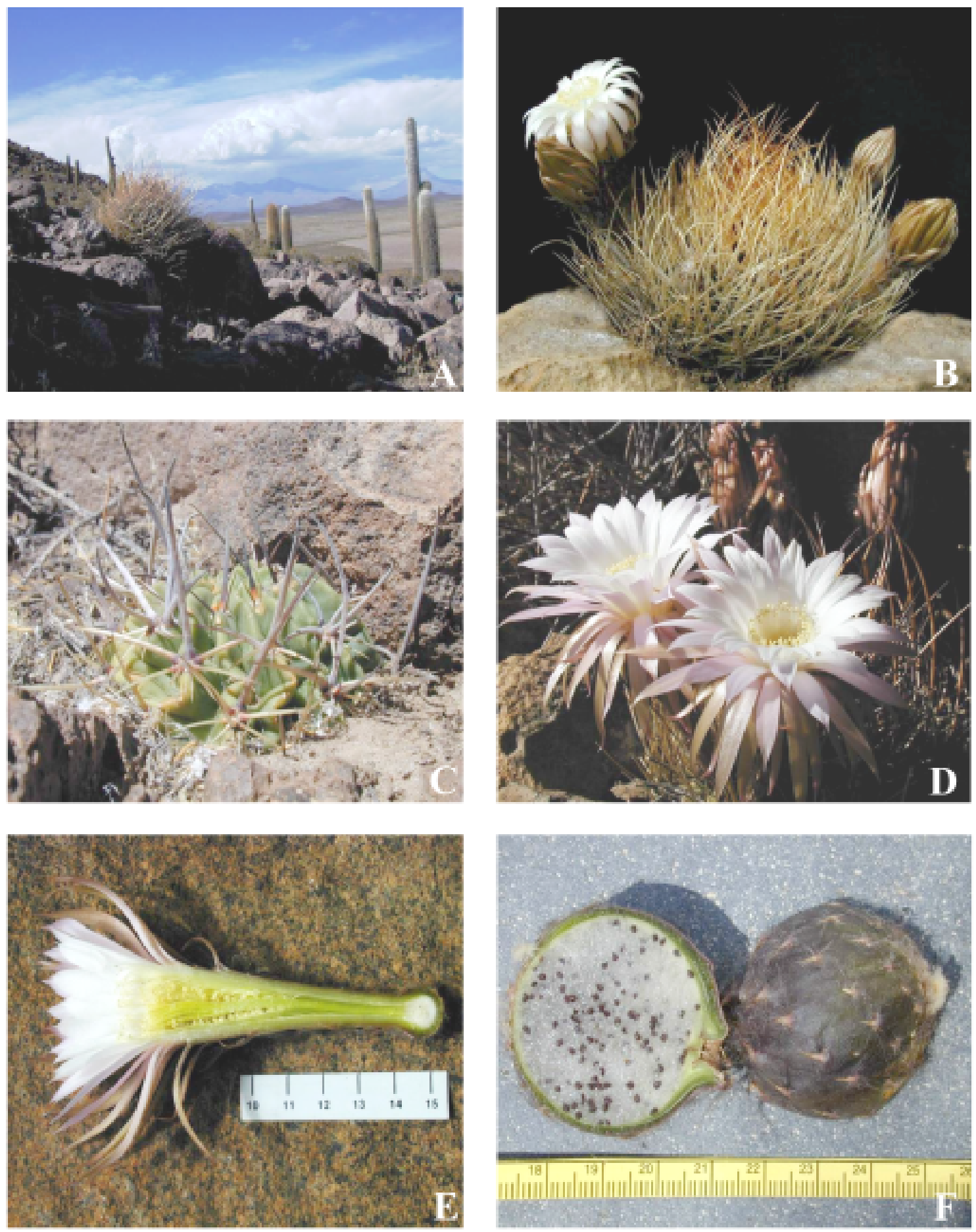

Figura 4. A. Lobivia ferox en su ambiente. B. Forma espinuda. C. Forma de espinas cortas. D. Flor rosada. E. Corte longitudinal de la flor. F. Corte longitudinal del fruto (fotos de A. Kirberg). 
Lobivia ferox, nuevo registro: PinTo R.

Descripción de flor y fruto: Flores laterales, grandes, de $11 \mathrm{~cm}$ de largo, de color rosado o blanco. Nacen de 6 a $8 \mathrm{~cm}$ del ápice, en la $3^{\circ}$ o $4^{\circ}$ areola formando una corona. Brotan de 3 a 7 flores por planta (Fig. 4 B y D). Individuos de espinas claras amarillentas presentan flores blancas e individuos de espinas oscuras grisáceas presen$\tan$ flores rosadas. Abren un solo día, sin perfume. Ovario muy peludo, tubo floral peludo, con escamas largas y carnosas de color café rosa. Pelos café blanquizcos o negruzcos. Botón muy peludo cuando inicia el desarrollo. Botón maduro con sépalos envolventes en espiral. Pétalos de $6 \mathrm{~mm}$ de ancho terminados en punta, 12 blancos interiores, 9 rosados pálido, 17 rosados más intenso. 18 sépalos color café rosa y 14 sépalos del mismo color curvados hacia fuera. Pistilo y estigma verde limón de $7 \mathrm{~cm}$ de largo que no sobrepasa los estambres. Estigma de 1,5 cm de largo presenta 10 divisiones. Estambres con filamentos blancos y anteras amarillo claro de $1 \mathrm{~mm}$, dispuestos en varias series a diferentes alturas (11). La serie de más arriba está separada $0,5 \mathrm{~cm}$ de la subsiguiente. El estigma llega hasta la penúltima serie de estambres (Fig. 4 E). Fruto esférico de 2,5 a $4,5 \mathrm{~cm}$ de diámetro por 3 a $5 \mathrm{~cm}$ de alto, de color verde oscuro con escamas verde rojizas y pelos en las axilas de éstas. De cáscara delgada (1,5 mm de grosor). Mantiene la flor seca pegada. En un fruto se contaron alrededor de 3.100 pequeñas semillas negras de $1,5 \mathrm{~mm}$ de alto por $1 \mathrm{~mm}$ de ancho, inmersas en una pulpa blanca (Fig. 4 F). Al cortar el fruto la pulpa se desprende entera fácilmente de la cáscara. Demora 1 a 2 meses en madurar. Al fruto cuando está maduro se le produce una perforación lateral cerca del ápice. Es muy difícil desprender el fruto que se encuentra protegido por las espinas, sólo es posible sacarlo junto con la areola. Plantas desde los $10 \mathrm{~cm}$ de diámetro producen flores. No todas las flores fructifican. Encontramos plantas hasta con 12 flores secas y plantas con hasta 6 frutos. Presenta botón en noviembre, flor en diciembre y fruto en enero y febrero.

\section{DISCUSION Y CONCLUSION}

Friedrich Ritter (1980) y Adriana Hoffmann (1989) relatan la historia de un cactus colec- tado por Knize en el norte de Chile (sin precisar localidad) y que se cultiva en Europa bajo el nombre de Lobivia chilensis. Ninguno de los dos pudo encontrarlo en sus expediciones. ¿Corresponderá a Lobivia ferox?

Rausch (1975) menciona el nomen nudum de L. chilensis como sinónimo de L. backebergii var. wrightiana Backeb. (p. 20), incluyendo el extremo norte de Chile (Tacora) en su distribución geográfica (p. 21) y una ilustración de ella (p. 23). En 1985 (p.136) menciona L. chilensis n.n. bajo L. wrightiana. Por otro lado, Ritter en 1980, vol. 3 p. 865 , menciona $L$. chilensis n.n. con duda, ya que no vio nada en Tacora. Al comparar un ejemplar de Panavinto y el corte de la flor con el dibujo de L. chilensis que Adriana Hoffmann hiciera llegar gentilmente, podemos encontrar bastantes semejanzas, sin embargo L. chilensis corresponde mejor a $L$. wrightiana Backbg. presente en Bolivia, por lo cual el hallazgo de L. ferox no resuelve el antiguo enigma.

Lobivia ferox es un cactus de grandes y hermosas flores, que produce un rico fruto comestible de un agradable sabor fresco, de pulpa blanca y semillas negras crujientes. Pareciera que las distintas formas de muchas espinas o pocas espinas tuvieran relación con la edad de la planta, no así las formas solitaria o agrupada. En algunos sitios es abundante, pero en otros, muy escaso. En sectores de mayor densidad hay presencia de juveniles. Las $3 / 4$ partes de la población está viva. Esto refleja un estado de conservación relativamente bueno, pero todavía no conocemos su rango latitudinal en territorio chileno. No hay que descartar que talvez sea la misma situación de la distribución de Echinopsis atacamensis, que en la I Región sólo está presente en Sierra Yarina y Sierra Chilani y talvez en Sierra Huailla. Este sector corresponde justamente a un triángulo que incursiona en territorio boliviano. Ambas especies $L$. ferox y E. atacamensis, son típicamente bolivianas.

El hallazgo de Lobivia ferox constituye un nuevo registro para la flora chilena y contribuye a ampliar el rango de distribución de esta especie hacia el oeste. A pesar de ser una especie nueva para la flora chilena, no hay ningún campesino aymara que no conozca el "sancave" y que no haya comido su fruto. 


\section{AGRADECIMIENTOS}

Agradezco al Dr. Beat Leuenberger del Museo Botánico de Berlín en Alemania y al profesor Clodomiro Marticorena de la Universidad de Concepción por las sugerencias y comentarios al manuscrito. Al Dr. Martin Lowry de Inglaterra, Dr. Roberto Kiesling de Argentina, Dr. Roberto Vásquez de Bolivia, Dr. Carlos Ostolaza de Perú y Adriana Hoffmann de Chile, a todos ellos agradezco su valiosa información.

\section{BIBLIOGRAFIA}

Backeberg, C. \& F. M., Knuth. 1936. Kaktus-ABC, Haandbog for fagfolk og amatorer, Nordisk, pp. $220-221$.

Backeberg, C. 1942. Neue Arten aus "Stachlige Wildnis". Repertorium Species in Novalum Regni Vegetabilis 51: 61-65.

Backeberg, C. 1943. Stachlige Wildnis. $80.000 \mathrm{~km}$ durch die Urwelt Americas. 2. Aufl. Neudamm und Berlin. 1 retrato, viii, 422 pp., 90 lám., 5 mapas y texto, 3 lám.

Brako L. \& J.L. Zarucchi. 1993. Catálogo de las Angiospermas y Gimnospermas del Perú. Monographs in Systematic Botany from the Missouri Botanical Garden, USA, 45: 1-1286.

Britton, N.L. \& J.L., Rose. 1922. The Cactaceae, 3. Carnegie Institution, Publ. N ${ }^{\circ} 248$, Washington.

Hoffmann, A. E. 1989. Cactáceas en la Flora Silvestre de Chile. Ed. Fundación Claudio Gay, Santiago. $272 \mathrm{pp}$.

Hunt, D. 1992. Cites Cactaceae Checklist. Royal Botanic Gardens Kew, 160 pp.

Kiesling, R, 1999. Les Cactées de Bolivie. Succulentes, numéro spécial, 3-48 pp.

Rausch, W. 1975, 1977. Lobivia I-II-III. Wien.

Rausch, W. 1986. Lobivia 85. Rüdolf Herzig, Wien.

RitTer, F. 1980. Kakteen in Südamerika Argentinien/ Bolivien Band 2: 375-856. Friedrich Ritter, Selbstverlag, Spangerberg.

http://www.med-phys.hull.ac.uk/Lobivia/Orig/ index.shtml Lobivia Names Database

Fecha de recepción: 01.07.02

Fecha de aceptación: 16.10.02

Fecha de publicación: Abril de 2003. 